\title{
Gradhiva
}

GRADHI

Revue d'anthropologie et d'histoire des arts

18 | 2013

Le monde selon l'Unesco

\section{Claude Lévi-Strauss, un parcours dans le siècle et Les Structures de l'esprit. Lévi-Strauss et les mythes}

Vincent Debaene

\section{OpenEdition}

Journals

Édition électronique

URL : http://journals.openedition.org/gradhiva/2758

DOI : 10.4000/gradhiva.2758

ISSN : 1760-849X

Éditeur

Musée du quai Branly Jacques Chirac

Édition imprimée

Date de publication : 1 décembre 2013

Pagination : 242-253

ISBN : 978-2-35744-072-2

ISSN : 0764-8928

Référence électronique

Vincent Debaene, "Claude Lévi-Strauss, un parcours dans le siècle et Les Structures de l'esprit. Lévi-

Strauss et les mythes », Gradhiva [En ligne], 18 | 2013, mis en ligne le 01 décembre 2016, consulté le 22 septembre 2020. URL : http://journals.openedition.org/gradhiva/2758 ; DOI : https://doi.org/10.4000/ gradhiva.2758

(c) musée du quai Branly 


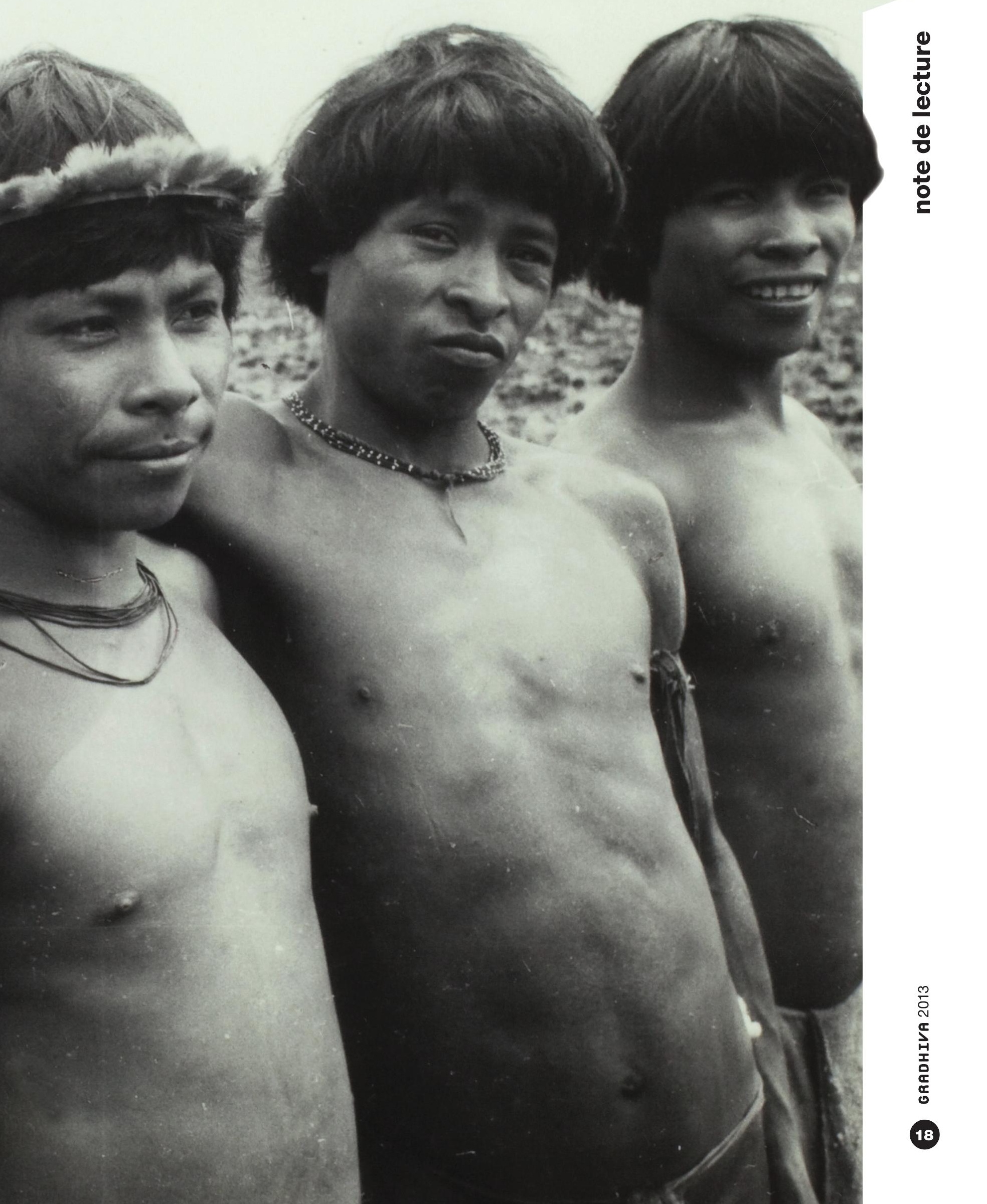




\title{
Claude Lévi-Strauss, un parcours dans le siècle et Les Structures de l'esprit. Lévi-Strauss et les mythes
}

\author{
par Vincent Debaene \\ Philippe Descola (dir.), Claude Lévi-Strauss, un parcours dans le siècle. \\ Paris, Odile Jacob ("Travaux du collège de France »), 2012. \\ Gildas Salmon, Les Structures de l'esprit. Lévi-Strauss et les mythes. \\ Paris, PUF («Pratiques théoriques»), 2013.
}

Lévi-Strauss, that's where it's at («Lévi-Strauss: c'est là que ça se passe»). Cette phrase, et d'autres semblables, entendue par Pierre Maranda lors d'un récent congrès d'anthropologie, est peut-être excessive mais que, depuis quelques années, quelque chose se passe autour de l'œuvre de Claude Lévi-Strauss, cela en revanche ne fait aucun doute - quelque chose que l'on aurait tout à fait tort de réduire à de simples effets de commémoration. Si besoin était, deux livres importants - mais très différents - viennent confirmer ce diagnostic: Claude Lévi-Strauss, un parcours dans le siècle, sous la direction de Philippe Descola, et Les Structures de l'esprit. Lévi-Strauss et les mythes de Gildas Salmon.

Alors que, après la mort de l'auteur de La Pensée sauvage, une anthropologie un peu paresseuse (parfois le fait d'autoproclamés structuralistes «repentis») se sentait autorisée à un facile «droit d'inventaire» et déclarait périmés le triangle culinaire, la formule canonique des mythes et autres prétendus «errements", voilà deux ouvrages qui, au contraire, redonnent à l'anthropologie de Lévi-Strauss toute son ambition théorique - mais qui le font de deux façons distinctes: I'un de l'intérieur de la discipline anthropologique, l'autre de l'extérieur.

Claude Lévi-Strauss, un parcours dans le siècle recueille les contributions au colloque organisé par Descola au Collège de France en novembre 2008 à l'occasion du centenaire de Lévi-Strauss. C'est un ouvrage remarquable en raison, d'abord, de son caractère intradisciplinaire. Au fond, parmi les très nombreux textes et études parus au moment du centenaire puis de la mort de Lévi-Strauss, et à l'exception de quelques articles isolés, il s'est trouvé peu de choses proprement anthropologiques. La plupart des numéros spéciaux publiés à cette occasion furent le fait de revues généralistes ou philosophiques ${ }^{\mathbf{1}}$, et si dans cette masse de contributions il s'est trouvé des travaux originaux et de grande qualité, l'immense majorité d'entre eux avait néanmoins une valeur biographique, historique, voire franchement spéculative. Ils visaient à situer l'œuvre dans son contexte historique ou à évaluer son retentissement dans le champ intellectuel au sens large, mais ne cherchaient pas à la relancer ou à en faire usage dans l'univers théorique où elle était née, à savoir au sein même des études anthropologiques ${ }^{2}$. Dans la note critique qu'il donna en 2008 à l'occasion de la parution du volume d'CEuvres dans la Bibliothèque de la Pléiade, Eduardo Viveiros de Castro, qui se réjouissait de ce " "deuxième printemps" de la littérature sur Lévi-Strauss», s'étonnait d'ailleurs que «l'héritage du structuralisme anthropologique» fût, «en France, mieux géré par la philosophie que par l'anthropologie ${ }^{3}$ ».
1. Au Cambridge Companion de 2009 et aux numéros spéciaux de Critique en 1999, des Archives de philosophie en 2003, des Temps modernes en 2004, des Cahiers de l'Herne en 2004, d'Esprit en 2004 et 2011, de Philosophie en 2008 , on peut ajouter les récents numéros des revues Europe et Yale French Studies (2013). II y a à cet égard une exception brésilienne puisque le numéro spécial de la Revista de antropologia de 1999 et le recueil Lévi-Strauss. Leitura Brasileiras (2008) se concentraient quant à eux sur l'héritage proprement anthropologique de LéviStrauss. Pour une tentative de synthèse des récentes relectures de ce dernier, je me permets de renvoyer à mon article "Lévi-Strauss aujourd'hui » dans Europe (n¹005-1006).

\begin{abstract}
2. En raison de sa portée philosophique ou de ses emprunts au monde de l'art et de la littérature, on sous-estime souvent le caractère fondamentalement disciplinaire du travail de LéviStrauss, dont les propositions théoriques les plus audacieuses et les plus marquantes sont toujours nées en réponse à de "vieilles questions " internes à la discipline anthropologique telle qu'elle s'est développée depuis Edward Tylor.
\end{abstract}

3. Eduardo Viveiros de Castro, "Claude Lévi-Strauss, CEuvres ", Gradhiva 8, 2008 : 133.
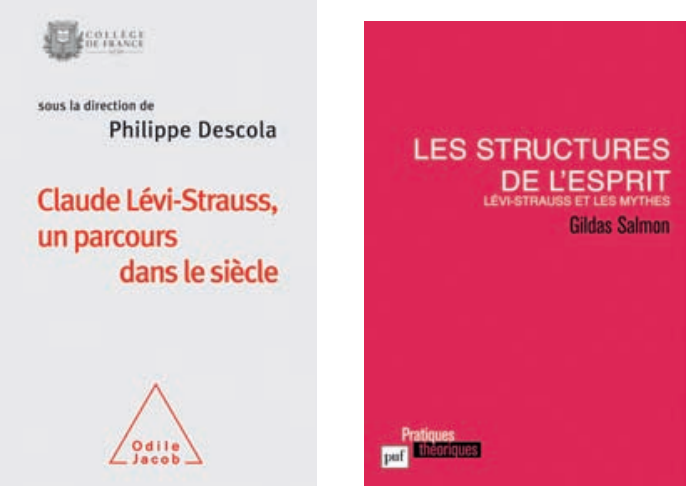
4. La seconde proposition est commentée également par Maurice Bloch et Marshall Sahlins dans leurs contributions respectives. Eux aussi se donnent pour tâche de la traduire à destination des anthropologues anglophones (p. 218 et 257).
Ce recueil vient en quelque sorte rectifier cet état de fait. À l'exception de la philosophe Claude Imbert (dont l'enseignement et la réflexion jouèrent un rôle essentiel dans le retour à Lévi-Strauss à partir des années 1990), tous les auteurs sont des anthropologues, parfois d'anciens étudiants de Lévi-Strauss, souvent des interlocuteurs de longue date (le regretté Luc de Heusch donne ici un article intitulé «Pouvoir et parenté en Afrique centrale », qui est sans doute un de ses derniers textes). Les travaux réunis dans ce volume permettent donc de comprendre le retentissement de la réflexion lévistraussienne dans les différentes spécialités d'une discipline anthropologique qui s'est considérablement développée mais aussi transformée et sectorisée depuis que Lévi-Strauss y fit ses premières armes avec sa «Contribution à l'étude de l'organisation sociale des Indiens bororo» en 1936.

Le volume est découpé en six parties thématiques («Terrains», «Parenté et mythologie», «Logique de la connaissance», etc.), mais dans les faits presque tous les articles sont conçus de la même façon: ils s'emparent d'une affirmation de Lévi-Strauss et se proposent de la tester, parfois de l'étendre ou de la varier, en dehors de son contexte théorique ou ethnographique d'origine - geste intellectuel caractéristique d'une pratique disciplinaire interne puisqu'il ne s'agit nullement de gloser ou de commenter les textes, mais d'évaluer l'efficacité ou l'opérativité d'une proposition dans un domaine d'études donné.

Par exemple, Maranda se saisit de deux des plus fameuses phrases de l'ouverture du Cru et le Cuit («Les mythes se pensent dans les hommes et à leur insu » et «Les mythes se pensent entre eux») et, dans une réponse imaginaire à Edmund Leach (à qui ces formules paraissaient tout simplement absurdes), tâche de comprendre ce qu'elles peuvent vouloir dire concrètement ${ }^{4}$. II propose donc de les mettre à l'épreuve dans un contexte ethnographique particulier, à propos du mythe d'origine de l'igname chez les Lau de Malaita (dans l'archipel des Salomon). Ce mythe d'origine, marqué dans toutes ses versions par la réversibilité de la métamorphose de l'igname en poisson, semble pouvoir être mis en rapport avec deux réalités sociales locales: l'institution des marchés côtiers comme lieu de transaction entre gens de la montagne et gens de la lagune, et le fait que ces marchés sont organisés et gérés par les femmes à l'exclusion des hommes. Maranda relate la réaction de ses interlocuteurs lau à son hypothèse interprétative (peut-on mettre en rapport ces trois réalités: mythe de l'igname/marchés/ initiation des femmes?). II s'interroge sur les mécanismes mentaux qui font que, la plupart du temps, la réponse est négative et sur les raisons de ce «cloisonnement» qui rendent le rapprochement entre ces trois faits sinon impossible, du moins difficilement pensable (si l'hypothèse de départ est valide, alors il faut en conclure que les mythes se pensent à l'insu des hommes). Maranda s'attarde également sur la forme prise par l'unique réponse positive, recueillie auprès d'un lettré (un liotoo), et sur les conditions de la réflexivité et de la métacognition, qu'il met en rapport avec la philosophie locale de la métaphore. II propose enfin une réflexion plus générale sur la circulation des mythes et leur transformation lorsqu'ils se déplacent, et sur les «modes cognitifs» qui font que les mêmes opérations logiques (synecdoques, métaphores, métonymies) fonctionnent parfois à l'insu et parfois au su de ceux en qui elles opèrent. 
Manuela Carneiro da Cunha mesure elle aussi la fécondité de la réflexion de Lévi-Strauss, en montrant comment la fameuse «idéologie bipartite » mise en évidence dans Histoire de lynx (essentiellement à propos de l'Amérique du Nord) se retrouve dans deux institutions - le compagnonnage et l'amitié formelle - telles qu'on les observe chez les Indiens krahó et canela (groupe gê). Dans ces deux cas, on retrouve «la coexistence d'un dualisme diamétral statique avec une formule dynamique introduite par un déséquilibre entre jumeaux». Carneiro da Cunha va même plus loin puisque, selon elle, ce double exemple illustre une forme particulière de "diffusion structurale", c'est-à-dire permet de démontrer la circulation interculturelle d'institutions ou de variantes mythiques, non pas en dépit mais en raison de leurs différences. À l'opposé du diffusionnisme classique, le concept essentiel de ce "diffusionnisme structuraliste" n'est pas l'emprunt à l'identique mais au contraire le "double retournement" à l'interface entre deux zones linguistiques ou culturelles (elle retrouve ici les récentes relectures de la formule canonique, par Maranda et Mauro Almeida en particulier).

Quoique dans une spécialité très éloignée, c'est une démarche semblable qui anime la réflexion de Carlo Severi, puisqu'il s'agit de tester la conception de l'œuvre d'art que Lévi-Strauss développe au début de La Pensée sauvage dans un domaine que, à première vue, celle-ci exclut: l'art non figuratif. Que faire en effet de l'hypothèse selon laquelle le «modèle réduit» offre «toujours et partout» «le type même de l'œuvre d'art » quand l'art a renoncé à toute prétention d'imitation? À partir de l'exemple de Vassily Kandinsky, Severi propose de montrer la fécondité d'une telle définition en étendant d'une part la notion de modèle (c'est «l'expérience de la forme", et non un objet naturel, qui est ici sujet de la représentation) et en montrant d'autre part que le travail de ce peintre s'inscrit dans une "série": comme les sculpteurs évoqués dans La Voie des masques, l'artiste russe «réplique à d'autres créateurs, récents ou passés, actuels ou virtuels" - et s'inscrit dans un espace défini par les différentes solutions plastiques offertes au "problème de la relation entre espace et couleur", auquel ont répondu à leur façon William Turner, Piet Mondrian ou Robert Delaunay.

De Heusch et Marshall Sahlins proposent quant à eux un regard rétrospectif sur l'ensemble de leurs travaux respectifs, en montrant comment ceux-ci furent sinon inspirés, en tout cas stimulés, par l'œuvre de LéviStrauss. Le premier revient sur son étude du statut du neveu utérin chez les Telela-Hamba et situe le système de parenté de ces derniers dans la cartographie dressée par les Structures élémentaires de la parenté, avant de proposer une solution à ce qui, en apparence, constitue une sorte de résistance de l'Afrique aux analyses structurales (peu de mythes mais une «foule de rites les plus divers»): «la mythologie explicite se réfugie très souvent, en Afrique, dans la "pseudo-histoire" ", tout particulièrement celle qui retrace l'origine de la royauté sacrée dont de Heusch montre les invariants symboliques, en dépit de la diversité des situations socioéconomiques. Sahlins inscrit quant à lui ses propres travaux en réaction à la proposition de La Pensée sauvage qui fait de l'ethnologie une «science des superstructures ": à l'aide d'exemples ethnographiques précis, il rétablit une perméabilité entre infra- et superstructures et caractérise sa réflexion comme une sorte "d'infrastructuralisme» qui montre comment les systèmes 
Danse du clan Paíwe. "... Le mort appartenalt à la moítí́ Cera; c'étaient done les Tugaré quí officiahent. Au centre de la place, une jonchée de feuillages figurait la tombe absente, flanquée à droi te et à gaúche par des faisceaux de flèches devant lesquels dés bols de nourriture avaient été disposés...Pendant les premières nuits nous avons assisté aux denses de divers clans tugaré $;$ ewoddo, danse de ceux du palmier; paíwe, danse de ceux du hérisson. Dans les deux cas, les danseurs étaient couverts de feuillage de la tête aux pleds et commi on ne voyait pas leur visage, celui-ci était imaginé plus haut, au niveau du diadème de plumes qui dominait le costu. me si bien qu'on prêtait involontairement aux personnages une taille démesurb̌e. Dans leurs mains, ils tenaient des hampes de palmes ou des bâtons ornés de feuilles. Il y avait deux sortes de danses. D'abord les danseurs se produisaient seuls, répartis en deux quadrilles se faisant face aux extrémités du terrain, courant l'un ver 1 fautre en oxiant "ho ! ho I" et tourbillonnant sur eux-mêmes jusqu'à ce qu'ils alent échangé leurs positions initiales. Plus tard, des femmes s'intercalaient entre les denseurs masculins et o'était alors une interminabl farandole qui se formait, avançant ou piótinant, conduite par des coryphées nus marchant à reculons et agitant leurs hochets tandis que d'autres hommes chantalent accroupis.".. (Tristes Tropiques).

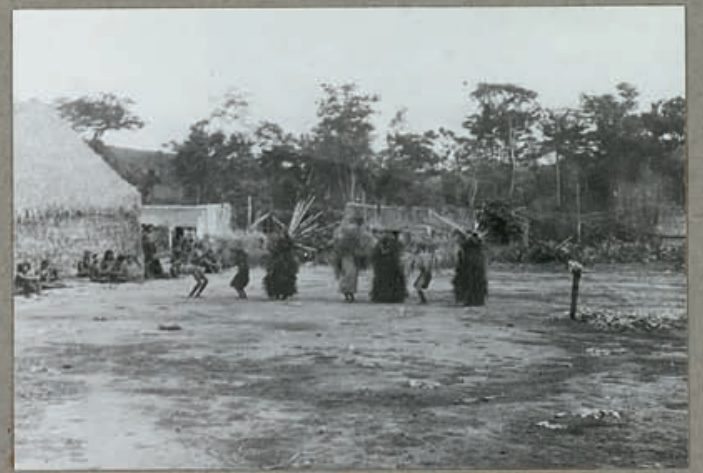

fig. 1

Claude Lévi-Strauss,

Danse du clan Paiwe, chez les Bororos, Mato Grosso,

Brésil, 1935-1936.

() musée du quai Branly. 


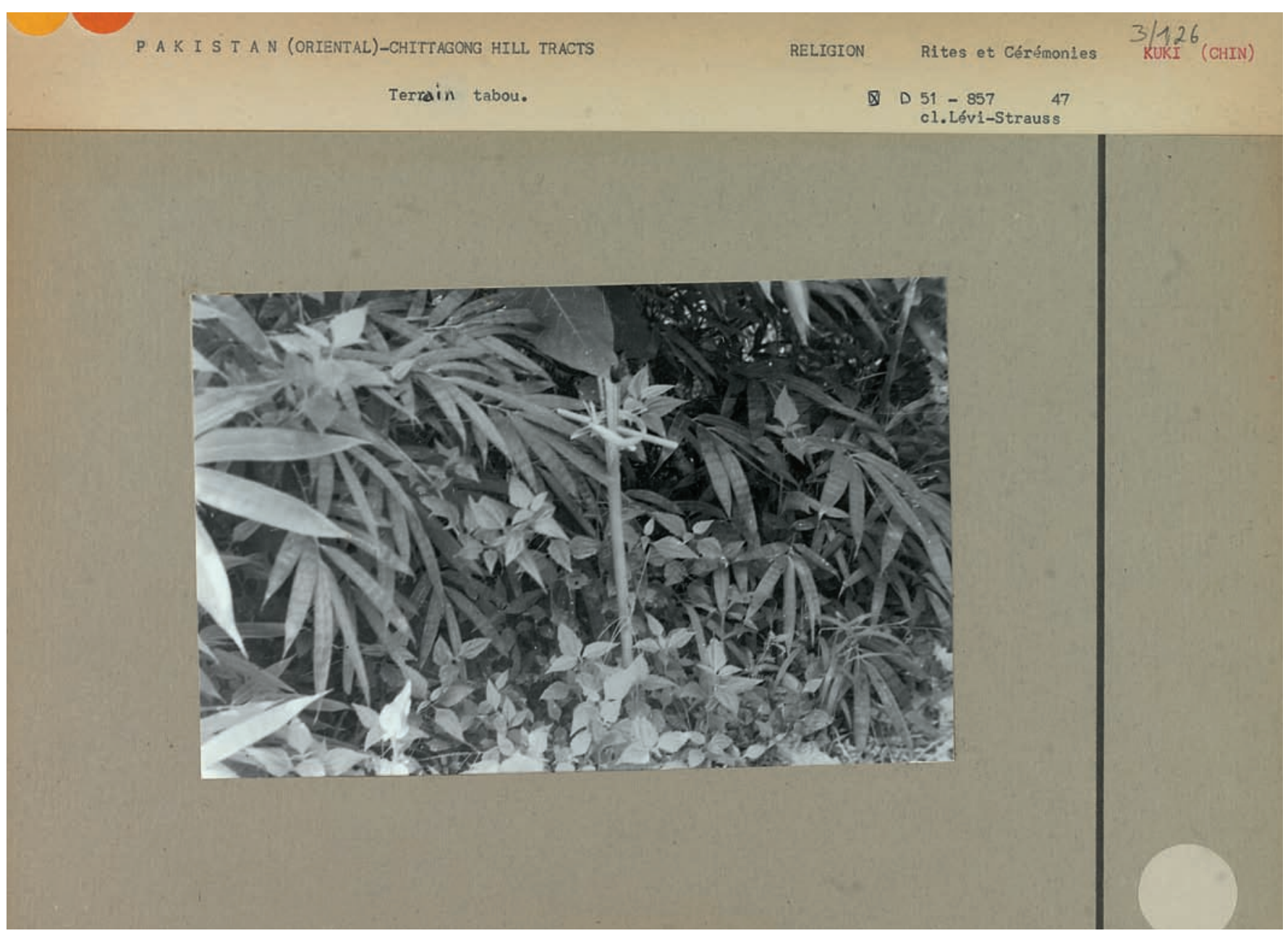

fig. 2

Claude Lévi-Strauss,

Terrain tabou, chez

les Kukis, Chittagong zila,

Bangladesh, 1951.

(C) musée du quai Branly. 
d'échanges de biens, loin d'être la source des représentations culturelles (comme le voulait le «matérialisme culturel» de Marvin Harris dans les années 1960), sont au contraire des systèmes «toujours déjà » symboliques de part en part.

Les démarches de Roberte Hamayon et Françoise Héritier sont comparables, mais plutôt que d'envisager l'effet de Lévi-Strauss sur une œuvre individuelle, elles s'attachent à comprendre son retentissement sur un domaine d'études dans son ensemble. La seconde montre que, en dépit de divergences internes parfois importantes, l'anthropologie de la parenté continue à travailler dans le cadre défini par Lévi-Strauss et sa réflexion sur la prohibition de l'inceste. La première propose une description très intéressante des usages de Lévi-Strauss dans un domaine - les études sibériennes - marqué par de fortes spécificités: l'impossibilité de travail sur le terrain pendant des décennies conduisit à travailler sur des sources datant pour l'essentiel du tournant du XIX siècle et à "accorder le présent ethnographique à la période présoviétique», les chercheurs trouvant un stimulant dans «la proposition lévi-straussienne de voir en tout fait, mot, geste, artefact... si minime soit-il, un "exercice de la pensée" ». Elle décrit également le rôle important joué par les réflexions de Lévi-Strauss dans l'analyse des rituels chamaniques et trouve, elle aussi, dans le contraste avec la réalité américaine un objet de réflexion: ici encore, la quasi-absence de la mythologie est l'occasion d'une comparaison très éclairante non pas, cette fois, avec la pseudo-histoire (comme dans la réflexion de De Heusch à propos du Congo ou du Rwanda) mais avec l'épopée qui par son contenu comme par son exécution rituelle «fonde la norme» chez de nombreux peuples sibériens.

Outre la réflexion épistémologique qu'Imbert consacre à ce qu'elle appelle le «second naturalisme» de Lévi-Strauss, trois contributions, enfin, choisissent une approche plus herméneutique, moins directement attachée à la pratique disciplinaire. Marie Mauzé propose une généalogie de la pensée du style de Lévi-Strauss et montre «le rapport paradigmatique entre l'ethnographie de la côte Nord-Ouest et l'analyse structurale». Maurice Bloch offre de restituer l'ambition profonde du projet lévi-straussien («étudier la nature humaine dans le cadre élargi de l'évolution ») par un double contraste à la fois à l'égard des fondateurs de la discipline (dont Lévi-Strauss refuse les postulats mais conserve l'ambition théorique) et à ses contemporains (dont il réprouve le particularisme mais retient la fidélité à la variété et à la complexité des réalités ethnographiques). Daniel Fabre, enfin, propose une réflexion très originale sur le judaïsme de Lévi-Strauss: il analyse «la refondation de l'appartenance» opérée par l'arrière-grand-père musicien, Isaac Strauss, qui, par sa pratique de collectionneur, invente la catégorie «d'art juif» et contribue à «une opération d'objectivation de la culture», objectivation qu'il faut «entendre inséparablement dans son sens concret - mise en objets - et dans son sens intellectuel». Ce faisant, Fabre apporte, à la suite de Victor Karady, une contribution importante à l'éclaircissement de cette énigme sociologique et historique, à savoir «le rôle joué par trois générations de juifs émancipés, de juifs non pratiquants ou même décidément athées, dans la création en Europe et en Amérique du Nord des sciences de la société et de la culture" (Émile Durkheim, Marcel 
Mauss, Franz Boas, etc.). Si la formule n'était pas si galvaudée, on parlerait volontiers des linéaments d'une anthropologie de l'anthropologue.

On a beaucoup dit que l'œuvre de Lévi-Strauss était remarquable par sa capacité à irriguer d'autres champs de la pensée, mais cet ouvrage collectif vient opportunément rappeler qu'elle a d'abord fondamentalement modifié les façons de faire et de penser au sein même de la discipline anthropologique, même si - seul petit regret - on aurait aimé quelques contributions plus prospectives, qui auraient envisagé certains usages présents, actuels ou potentiels, de Lévi-Strauss dans les grands chantiers contemporains de l'anthropologie. Les travaux de Descola (qui, pour l'occasion, s'est cantonné au rôle de maître de cérémonie) et de ses interlocuteurs, ou les développements consécutifs à ce qui est de plus en plus identifié comme un «tournant ontologique » en anthropologie, auraient sans doute fourni des exemples intéressants.

L'ouvrage de Salmon, Les Structures de l'esprit, se situe à la fois à l'opposé et dans la continuité de ce recueil. À l'opposé parce qu'il s'agit d'un ouvrage de philosophie et non d'anthropologie, mais dans la continuité puisque Salmon lit l'œuvre de Lévi-Strauss non comme celle d'un philosophe ou d'un penseur, mais bien comme celle d'un savant ${ }^{5}$. II s'agit de comprendre et mesurer l'événement qu'a constitué la réflexion de LéviStrauss pour l'ensemble des sciences humaines et au-delà, c'est-à-dire de comprendre d'une part la radicale nouveauté des analyses lévi-straussiennes, d'autre part de saisir l'effet de réorganisation qu'elles ont produit sur le champ du savoir. Le travail herméneutique de compréhension de l'œuvre s'appuie sur une démarche archéologique qui reconstitue les problèmes et les lignes de partage qui traversaient les disciplines avec lesquelles Lévi-Strauss était en dialogue au moment de la genèse de l'anthropologie structurale: linguistique, phonologie, anthropologie, psychanalyse, philologie, biologie 6 .

La thèse essentielle du livre est simple: Lévi-Strauss a redéfini l'anthropologie, et il l'a fait en redéfinissant le comparatisme. L'anthropologie structurale a été élaborée en réponse à une crise historique: elle est une tentative pour rendre à nouveau possible la comparaison interculturelle alors que la discipline était menacée d'éclatement entre l'accumulation de données ethnographiques de plus en plus dispersées et des synthèses comparatives de plus en plus fragiles. En posant que la comparaison doit reposer non sur l'inventaire des ressemblances perçues par l'observateur, mais sur la systématicité des différences entre cultures et sociétés, LéviStrauss a modifié "la manière même de construire les comparables". De sorte que le structuralisme n'est pas une nouvelle théorie anthropologique visant à «expliquer» les faits culturels et sociaux (théorie qui aurait succédé à l'évolutionnisme, au fonctionnalisme, etc.), il constitue bien une nouvelle façon de déterminer ces mêmes faits ${ }^{7}$. Du même coup, sont repensés les rapports entre ethnographie et anthropologie: loin de précéder la comparaison anthropologique (selon la classique division du travail intellectuel qui voudrait que l'anthropologue synthétise les données recueillies par d'autres), l'ethnographie est au contraire conditionnée par le projet anthropologique comparatiste.
5. De fait, de nombreux développements de son livre entrent en résonance avec les réflexions du recueil précédemment évoqué, en particulier concernant la question de la diffusion structurale à laquelle il consacre un chapitre, ou l'idée que les mythes "se pensent entre eux".

6. Dans deux passages très intéressants (à la fin de l'introduction et au début de la conclusion), Salmon situe sa propre démarche d'histoire conceptuelle en montrant ce qu'elle doit à l'archéologie foucaldienne et comment elle s'en distingue.

7. Voir cette remarque de Lévi-Strauss dans une discussion des travaux de Robert Lowie: "Même pour le structuraliste, cette question [quels sont les faits ?] est la première à laquelle il faille répondre, et [...] elle commande toutes les autres. " (Anthropologie structurale, Paris, Plon, 1958: 340). 


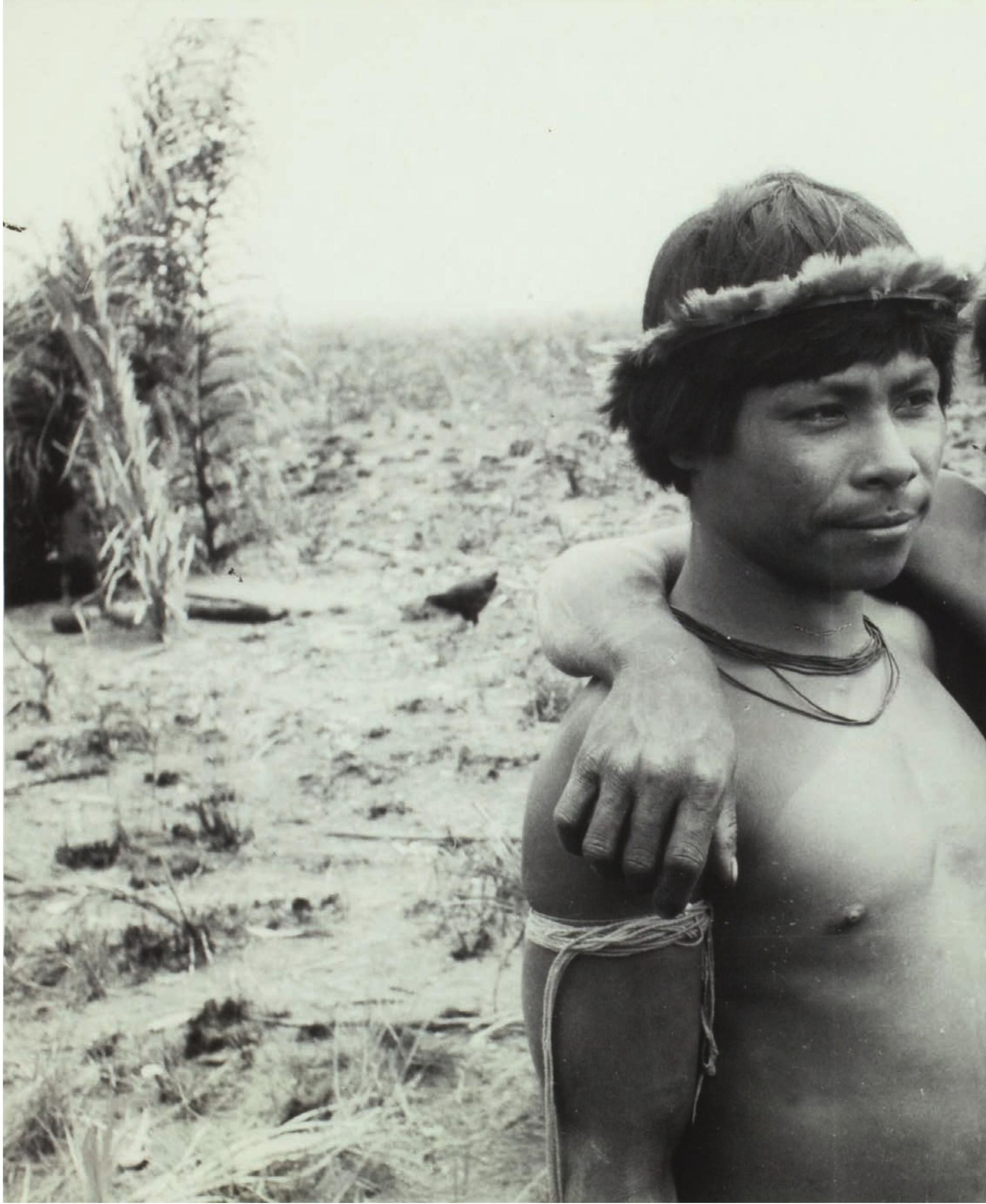




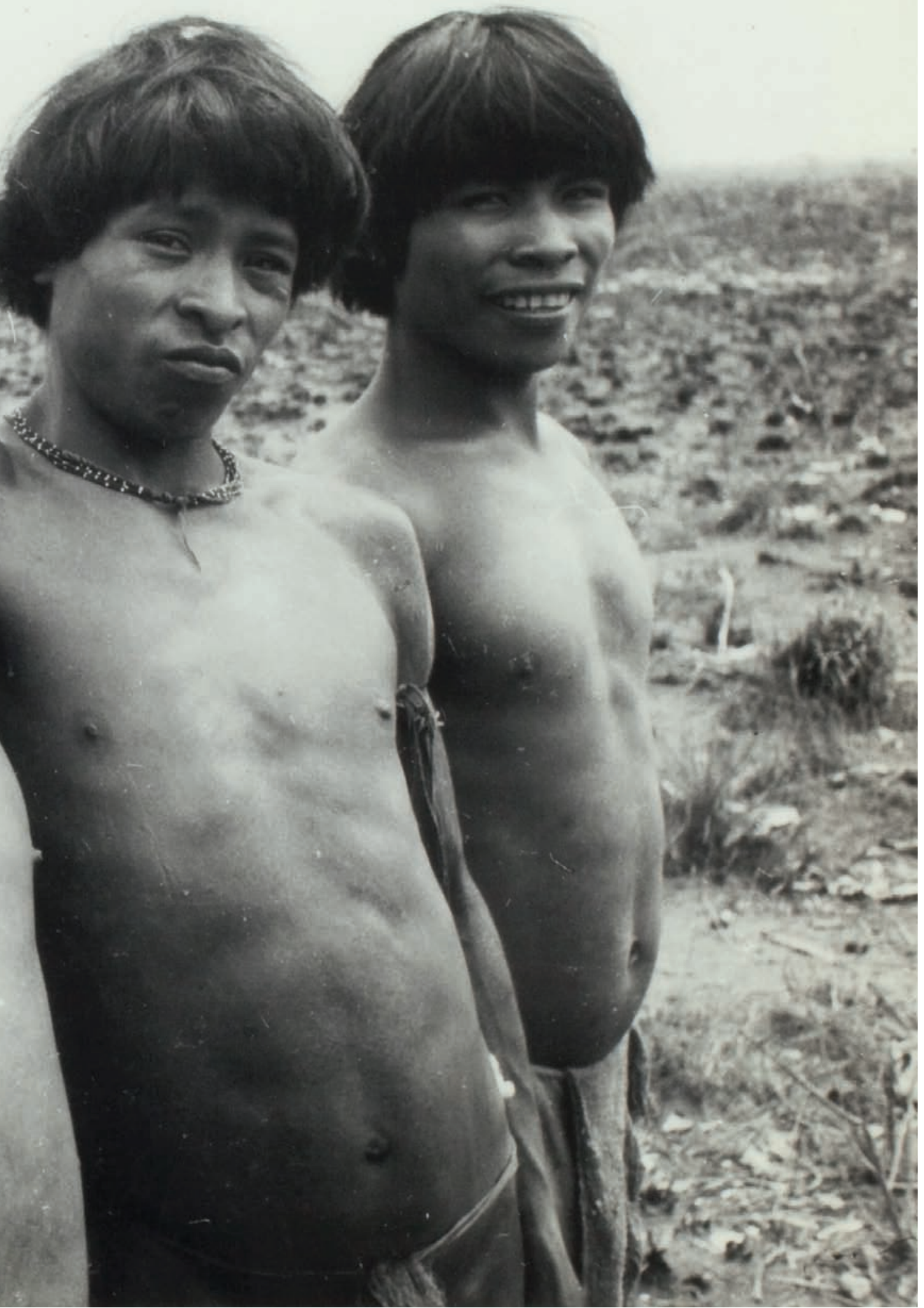

page 242 et ci-contre

fig. 3

Claude Lévi-Strauss,

Les beaux-frères, chez

les Nambicuaras, Brésil, v. 1938

○ musée du quai Branly. 
8. L'ouvrage considère en effet surtout la "seconde" anthropologie structurale. À la suite d'autres auteurs, Salmon insiste de façon très convaincante sur la rupture théorique entre ce qu'il appelle le "structuro-fonctionnalisme " des Structures élémentaires de la parenté et le structuralisme transformationnel des

Mythologiques.
La démonstration conduit Salmon à de très nombreuses propositions théoriques passionnantes, mais retenons les deux principales: la notion centrale de l'anthropologie structurale n'est pas celle de structure, mais de transformation; les relations de transformation que l'anthropologie peut identifier entre deux mythes ou deux rites de populations voisines correspondent aux opérations mentales réelles à l'origine de ces institutions: «Les structures de l'anthropologie lévi-straussiennes sont [...] celles de l'esprit humain. "Salmon souligne avec force qu'on ne peut saisir la portée véritable de l'anthropologie structurale qu'à la condition de maintenir cette «hypothèse psychologique extrêmement hardie» touchant la nature de l'esprit humain et son fonctionnement: les institutions sont produites à travers des procédures objectives de distinction, conséquences ellesmêmes d'opérations mentales qui ne sont analysables que parce qu'elles sont homologues de celles de l'anthropologue. Sans cette hypothèse d'une homogénéité entre les opérations de la pensée scientifique et celles de la pensée symbolique, non seulement on ne pourrait rendre compte de la systématicité avérée des relations entre variantes mythiques ou formes d'organisation sociale, mais la démarche anthropologique elle-même ne pourrait sortir du soupçon d'extériorité et d'artifice par rapport aux formes de pensée qu'elle prend pour objet. La théorie sémiologique de l'esprit humain n'est pas un supplément de l'anthropologie structurale qu'on serait libre ou non d'accepter comme un article de foi en sus des analyses ethnographiques plus positives, elle en est la condition.

Ce livre propose donc de reconstruire l'histoire des problèmes qui, explicitement ou souterrainement, organisaient différents champs disciplinaires (au sens très large) à l'orée de l'invention de l'analyse structurale des mythes $^{\mathbf{8}}$. La première partie porte sur la philologie et la mythologie comparée; la deuxième sur l'intertextualité mythique et les procédures d'interprétation; la troisième sur la pensée symbolique et la théorie de l'esprit. Dans ces trois domaines, l'analyse structurale a provoqué «des réorganisations internes» qui ont conduit à «une mutation profonde du savoir anthropologique».

On lit cet ouvrage avec un sentiment d'étonnement devant ce qui est pourtant familier. On y retrouve certes les noms connus qu'on attend de toute histoire du structuralisme: Ferdinand de Saussure, Roman Jakobson, Georges Dumézil, Marcel Mauss. Mais à ces noms s'en ajoutent d'autres, moins souvent cités mais essentiels à la compréhension du projet structuraliste: Edward Tylor, Max Müller, Marcel Granet, D'Arcy Thompson, Sigmund Freud - et surtout cette généalogie est complètement réorganisée. Granet apparaît ici non pour son rôle de précurseur dans l'analyse de la parenté, mais pour la nouveauté de sa perspective sur le discours légendaire. Le rapport de Lévi-Strauss à Freud est complètement revisité, loin des critiques parfois hâtives de La Potière jalouse. Les liens avec les travaux de Jakobson sont éclairés, mais la phonologie y occupe une place moins centrale que l'analyse du folklore. Ce travail archéologique est également l'occasion de quelques mises au point concernant certains débats théoriques importants postérieurs à l'avènement public de l'anthropologie structurale - avec Dan Sperber et l'anthropologie cognitive en particulier. 
On est à mille lieues de I'histoire cancanière du structuralisme comme mode intellectuelle; il est assez peu question de Michel Foucault (sinon au titre de référence épistémologique), à peine de Jacques Lacan, et pas du tout de Roland Barthes. On est également très loin du récit scolaire de filiation qui va de la linguistique à la phonologie puis à l'anthropologie par transferts successifs de méthode; c'est tout un univers théorique qui est cartographié à nouveaux frais, non à partir des "écoles de pensée", mais de problèmes qui traversent le siècle et les frontières disciplinaires - et dont on s'aperçoit d'un coup que l'anthropologie, la philosophie ou I'histoire des sciences contemporaines sont les héritières. Ce livre est donc une histoire théorique des sciences humaines au $x x^{e}$ siècle ainsi qu'un ouvrage sur notre présent et sur ses conditions.

II faut enfin souligner la maîtrise par Salmon des concepts, des problème et de leur histoire dans des domaines disciplinaires très variés (de la philologie à la biologie, de la psychanalyse à l'histoire des sciences). Il propose par ailleurs une vue d'ensemble sur l'œuvre de Lévi-Strauss (pourtant particulièrement touffue et complexe) d'une très grande clarté et d'une très grande fermeté. On sort de cette lecture souvent impressionné, et parfois franchement ébloui. Et qu'une synthèse si lumineuse tienne en moins de 300 pages ne fait que renforcer ce sentiment. 